\title{
Malignant Neuroleptic Syndrome following Deep Brain Stimulation Surgery of Globus Pallidus Pars Internus in Cerebral Palsy
}

\author{
Jae Meen Lee, M.D. ${ }^{1}$, Sun Ha Paek, M.D., Ph.D. ${ }^{1}$, Hye Ran Park, M.D. ${ }^{1}$, Kang Hee Lee, M.D. ${ }^{2}$, \\ Chae Won Shin, M.D. ${ }^{3}$, Hye Young Park, M.D. ${ }^{3}$, Hee Pyoung Park, M.D., Ph.D. ${ }^{2}$, Dong Gyu Kim, M.D., Ph.D. ${ }^{1}$, \\ and Beom Seok Jeon, M.D., Ph.D. ${ }^{3}$
}

${ }^{1}$ Department of Neurosurgery, ${ }^{2}$ Department of Anesthesiology, ${ }^{3}$ Department of Neurology, Seoul National University Hospital, Seoul, Korea

\begin{abstract}
Neuroleptic malignant syndrome (NMS) is a rare but potentially lethal outcome caused by sudden discontinuation or dose reduction of dopaminergic agents. We report an extremely rare case of NMS after deep brain stimulation (DBS) surgery in a cerebral palsy (CP) patient without the withdrawal of dopaminergic agents. A 19-year-old girl with CP was admitted for DBS due to medically refractory dystonia and rigidity. Dopaminergic agents were not stopped preoperatively. DBS was performed uneventfully under monitored anesthesia. Dopaminergic medication was continued during the postoperative period. She manifested spasticity and muscle rigidity, and was high fever resistant to anti-pyretic drugs at $2 \mathrm{~h}$ postoperative. At postoperative $20 \mathrm{~h}$, she suffered cardiac arrest and expired, despite vigorous cardiopulmonary resuscitation. NMS should be considered for hyperthermia and severe spasticity in CP patients after DBS surgery, irrespective of continued dopaminergic medication.
\end{abstract}

Key Words: cerebral palsy; deep brain stimulation; neuroleptic malignant syndrome.

Cerebral palsy (CP) is marked by motor disturbances along with abnormal movements and postures that result from damage to the developing brain.[1] Management of severe dystonia in children with congenital CP is usually difficult. Treatment options include physical modalities, dopaminergic drugs, anti-cholinergic drugs or botulinum toxin muscle relaxants.[2,3] Deep brain stimulation (DBS) has been found to be safe and effective in young patients with dystonia and spasticity of CP.[1,4] In DBS, those drugs are transiently stopped prior to the procedure for intraoperative microelectrode recording (MER). Neuroleptic malignant syndrome (NMS) is a rare but potentially lethally morbid, with a mortality rate between 10\% and 50\%.[5] It is marked by high fever, severe rigidity, autonomic instability, and an altered level of consciousness. [5,6] NMS can be caused by a sudden discontinuation or dose reduction of dopaminergic agents.[5-7] However, there is no report regarding a case showing NMS, despite scheduled medication of a dopaminergic agent without its dose reduction during the perioperative period. Herein, we report an extremely rare case of NMS after DBS surgery in a CP patient without a dose reduction of dopaminergic agents.

Received on September 8, 2015 Revised on November 20, 2015 Accepted on December 22, 2015

Correspondence to: Sun Ha Paek, Department of Neurosurgery, Seoul National University Hospital, 101 Daehak-ro, Jongno-gu, Seoul 03080, Korea

Tel: +82-2-2072-3993, Fax: +82-2-744-8459

E-mail: paeksh@snu.ac.kr

${ }^{*}$ No potential conflict of interest relevant to this article was reported.

\section{Case Report}

A 19-year-old girl with history of CP was scheduled for DBS of the globus pallidus pars internus (GPi) bilaterally due to medically refractory dystonia and rigidity . Her medication 
consisted of $300 \mathrm{mg} /$ day levodopa, $2 \mathrm{mg}$ /day diazepam, and $60 \mathrm{mg}$ /day baclofen. It was not necessary to gain clinical information by intraoperative stimulation during surgery because the operation was carried out under monitored anesthesia care (MAC) with propofol and continuous infusion of remifentanil. So she took routine medication in the morning of operation day. During the DBS prcedure, she was lightly sedated with dose adjustment of propofol and remifentanil to maintain bispectral index of $60-80$. The procedure was uneventful, with an implantation of DBS electrodes in the GPi bilaterally (Medtronic 3387 electrodes; Medtronic, Minneapolis, MN, USA). A postoperative brain computer tomography showed no intracranial abnormality and confirmed optimal lead placement (Fig. 1). The patient initially showed a normal awakening and normal laboratory findings

Table 1. Laboratory findings immediately after surgery

\begin{tabular}{lcc}
\hline Test & Value & Normal value \\
\hline Blood urea nitrogen $(\mathrm{mg} / \mathrm{dL})$ & 11 & $10-26$ \\
Creatinine $(\mathrm{mg} / \mathrm{dL})$ & 0.53 & $0.7-1.4$ \\
Sodium $(\mathrm{mmol} / \mathrm{L})$ & 142 & $135-145$ \\
Potasium $(\mathrm{mmol} / \mathrm{L})$ & 3.6 & $3.5-5.5$ \\
Aspartate aminotransferase $(\mathrm{U} / \mathrm{L})$ & 19 & $15-37$ \\
White blood cell count $\left(\times 10^{3} / \mu \mathrm{L}\right)$ & 9.73 & $4.0-10.0$ \\
$\mathrm{pH}$ & 7.28 & $7.36-7.46$ \\
$\mathrm{PCO}_{2}(\mathrm{mmHg})$ & 52.5 & $32-46$ \\
$\mathrm{HCO}_{3}(\mathrm{mmol} / \mathrm{L})$ & 22.4 & $21-29$ \\
\hline
\end{tabular}
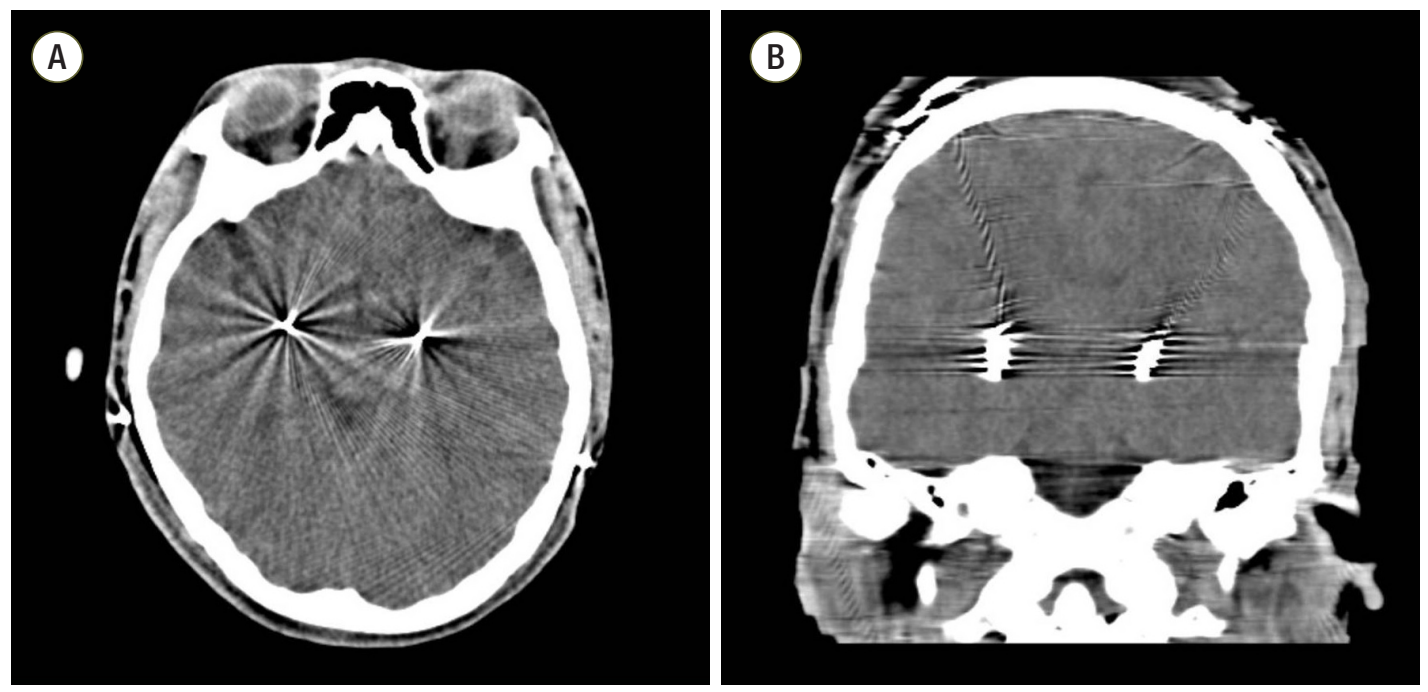

Fig. 1. Axial (A) and coronal (B) postoperative computed tomography images. There was no intracranial abnormality and electrode leads were in the proper positions. except mild respiratory acidosis (Table 1). However, on postoperative $2 \mathrm{~h}$, she manifested spasticity and muscle rigidity and took her medications, such as levodopa to relieve these symptoms. At that time, she had a high fever $\left(40.6{ }^{\circ} \mathrm{C}\right)$ resistant to anti-pyretic drugs (propacetamol $1 \mathrm{~g}$ ). We suspected that hyperthermia was caused by postoperative atelectasis, but chest X-ray showed no significant abnormality except postoperative ileus (Fig. 2). On postoperative $16 \mathrm{~h}$, her general condition worsened convulsively despite aggres-

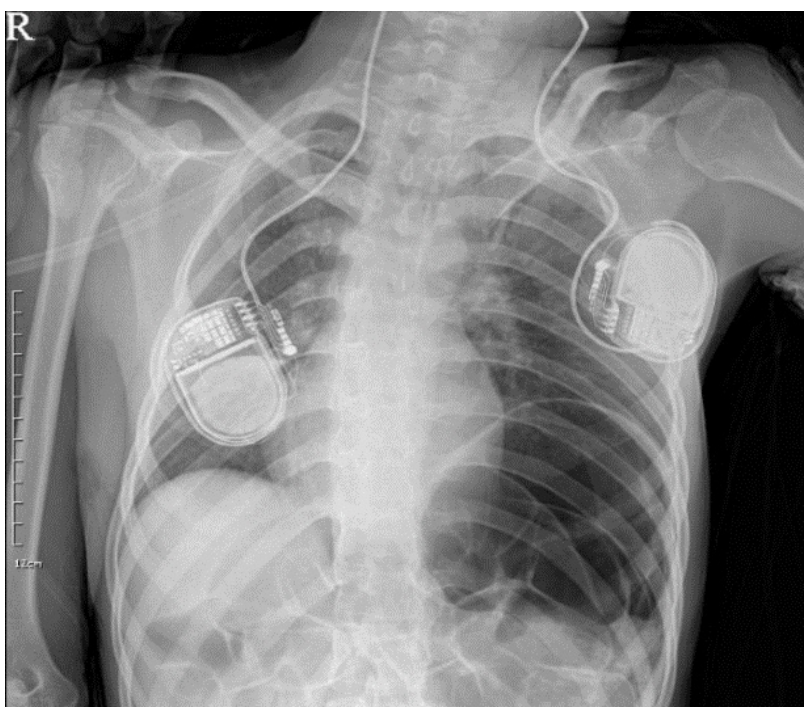

Fig. 2. An X-ray image shows no significant chest abnormality, however it does reveal abdominal air fluid levels suggesting ileus. 
sive care. She developed severe rigidity, tachycardia (heart rate: 170 beats $/ \mathrm{min}$ ), hyperventilation (respiration rate: 30 frequencies/min), and agitation. Her temperature had risen to $42^{\circ} \mathrm{C}$, which was refractory to copious cooling treatment such as surface tepid massage, and her blood pressure had increased to $149 / 77 \mathrm{mmHg}$ (Fig. 3).

On postoperative $20 \mathrm{~h}$, she fell rapidly into a coma and showed bilateral pupil fixation (Glasgow Coma Scale: 3). Immediately thereafter, she suffered cardiac arrest, We then started cardiopulmonary resuscitation (CPR) on her. A subsequent laboratory investigation showed leukocytosis (leukocyte count, $19.3 \times 10^{9} / \mathrm{L}$ ) with a shift to the left (Table 2). The

Table 2. Laboratory findings during cardiopulmonary resuscitation

\begin{tabular}{lcc}
\hline Test & Value & Normal value \\
\hline Blood urea nitrogen $(\mathrm{mg} / \mathrm{dL})$ & 36 & $10-26$ \\
Creatinine $(\mathrm{mg} / \mathrm{dL})$ & 1.93 & $0.7-1.4$ \\
\hline Sodium $(\mathrm{mmol} / \mathrm{L})$ & 158 & $135-145$ \\
\hline Potasium $(\mathrm{mmol} / \mathrm{L})$ & 6.4 & $3.5-5.5$ \\
Aspartate aminotransferase $(\mathrm{U} / \mathrm{L})$ & 767 & $15-37$ \\
White blood cell count $\left(\times 10^{3} / \mu \mathrm{L}\right)$ & 19.3 & $4.0-10.0$ \\
\hline $\mathrm{pH}$ & 7.13 & $7.36-7.46$ \\
$\mathrm{PCO}_{2}(\mathrm{mmHg})$ & 42.0 & $32-46$ \\
$\mathrm{HCO}_{3}(\mathrm{mmol} / \mathrm{L})$ & 11.9 & $21-29$ \\
$\mathrm{Creatine}_{\mathrm{Ninase}}(\mathrm{IU} / \mathrm{L})$ & 50038 & $20-270$ \\
\hline
\end{tabular}

serum creatine kinase (CK) level was markedly elevated to 50,038 IU/L. She also had high liver transaminases (serum glutamic-oxaloacetic transminase: $767 \mathrm{IU} / \mathrm{L}$, serum glutamic-pyruvic transminase: $116 \mathrm{IU} / \mathrm{L}$ ), hyperkalemia (serum potassium level: $6.4 \mathrm{mmol} / \mathrm{L}$ ) and metabolic acidosis on blood gas analysis (pH 7.13, $\mathrm{PCO}_{2} 42.0 \mathrm{mmHg}, \mathrm{HCO}_{3} 11.9$ $\mathrm{mmol} / \mathrm{L}$ ). We tried to revive her with $\mathrm{CPR}$ for 50 minutes, but failed to resuscitate her.

\section{Discussion}

This is the first case report of NMS related to the DBS in a $\mathrm{CP}$ patient without a dose reduction of dopaminergic agents during perioperative period.

NMS is a rare, fatal, and adverse reaction to neuroleptic drugs.[8] This could be triggered by the introduction of dopaminergic antagonist drugs (e.g. haloperidol) or the withdrawal of dopaminergic agonists (e.g. levodopa). Due to the association of NMS with dopaminergic agents, NMS is hypothesized to be related to a dopaminergic blockade in the hypothalamus. A defined diagnosis of NMS requires both essential features (hyperthermia and severe rigidity) and associated symptoms and signs (elevation of CK level, tachycardia, and altered mental status), which are listed in the Diagnostic and Statistical Manual of Mental Disorders.

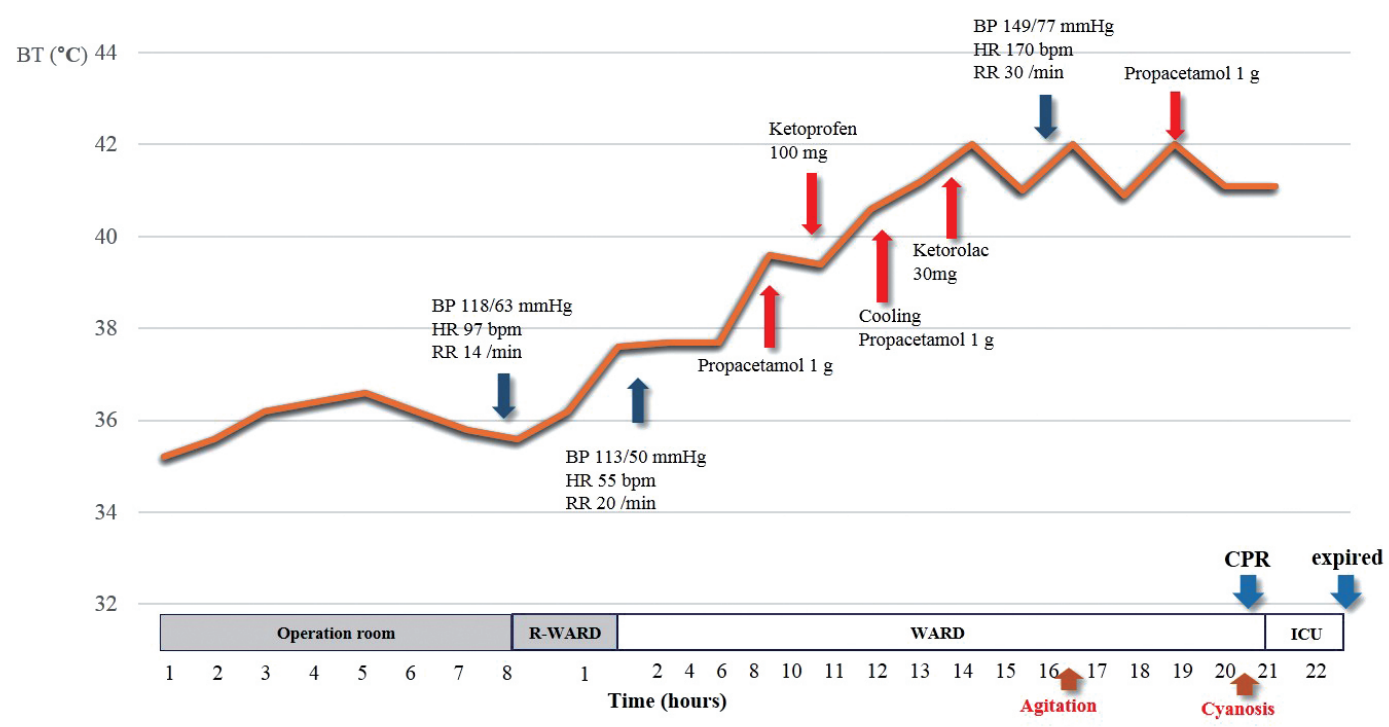

Fig. 3. A schematic illustration of changes in body temperature and the management of hyperthermia during the perioperative period. BT: body temperature; BP: blood pressure; HR: heart rate; RR: raspiration rate; R-ward: recovery ward; CPR: cardiopulmonary resuscitation; ICU: intensive care unit. 
$[8,9]$ A life-threatening complication of NMS is rhabdomyolysis, which is evidenced by an extreme elevation of the CK levels, myoglobinuria and acute renal failure.[10] Treatment of NMS is the rapid withdrawal of neuroleptic agents or replacement of dopaminergic agonists depending on early clinical recognition along with supportive care, which includes temperature control, rehydration, alkalization of urine to prevent renal failure, and repair of electrolyte balance.[5] In addition, bromocriptine (a dopaminergic agonist), amantadine (a dopaminergic agonist and a cholinergic antagonist), and dantrolene (a direct-acting skeletal muscle relaxant) can be also used.

Dopaminergic agents such as levodopa, are usually used in patients with Parkinson's disease (PD). It also has been used in cases with dystonic symptoms related to CP.[11] In these days, DBS has been used as a major therapeutic modality for movement disorders, such as PD, essential tremor and dystonia.[12,13] In particlar, DBS in GPi has been reported to be significantly useful in patients with dystonia.[14] Vidalihet et al.[1] showed that bilateral DBS also resulted in the improvement of motor symptoms and functional disability in CP patients with dystonia over a year.

The standard procedure of DBS is to use local anesthesia for intraoperative MER. This method allows intraoperative evaluation of the stimulation-induced improvement. [15] Thus, discontinuation of dopaminergic medication is, in clinical practice, common prior to DBS surgery, which can induce NMS in a previous study.[16] In our study, we performed the procedure under MAC. Therefore, her medications were not stopped preoperatively because we would not awaken the patient during the operation to evaluate stimulation-induced improvement. Moreover, dopaminergic medication was continued and anti-psychotic agents that have anti-dopaminergic effects were not administered during postoperative period. Nevertheless, this case report shows that symptoms and signs of NMS can be presented without reducing or discontinuing the drug. We did not explain the exact reason why NMS occurred in this patient postoperatively. A possible explanation is that DBS surgery per se might disrupt the absorption of dopaminergic drug by altering bowel movement and subsequently resulting in low blood concentration of dopaminergic agents. A previous study demonstrated that NMS resulted from a decreased intestinal absorption of dopaminergic agents in a patient with ileus.[17]

There are some diseases showing clinical manifestations similar with NMS. We can easily distinguish NMS from malignant hyperthermia because of different triggering agents. Succinylcholine and inhalation agents are triggering agents for malignant hyperthermia while neuroleptic agents for NMS. Status epilepticus rarely causes high fever. Encephalitis also causes an elevation in body temperature, but the peak value of fever is lower in encephalitis than in NMS. Moreover, rhabdomyolysis is rarely shown in encephalitis. $[18,19]$

In conclusion, clinicians should consider NMS as a differential diagnosis when high fever refractory to conventional treatments, spasticity, and severe rigidity following the DBS surgery are manifested in a CP patient, who take dopaminergic medication on schedule time during the perioperative period. Early diagnosis and rapid aggressive management for NMS should be needed to prevent the regrettable result.

\section{References}

1) Vidailhet M, Yelnik J, Lagrange C, Fraix V, Grabli D, Thobois S, et al: Bilateral pallidal deep brain stimulation for the treatment of patients with dystonia-choreoathetosis cerebral palsy: a prospective pilot study. Lancet Neurol 2009; 8: 709-17.

2) Jankovic J: Dystonia: medical therapy and botulinum toxin. Adv Neurol 2004; 94: 275-86.

3) Bhidayasiri R: Dystonia: genetics and treatment update. Neurologist 2006; 12: 74-85.

4) Kim AR, Chang JW, Chang WS, Park ES, Cho SR: Two-year outcomes of deep brain stimulation in adults with cerebral palsy. Ann Rehabil Med 2014; 38: 20917.

5) Adnet P, Lestavel P, Krivosic-Horber R: Neuroleptic malignant syndrome. Br J Anaesth 2000; 85: 129-35.

6) Newman EJ, Grosset DG, Kennedy PG: The parkinsonism-hyperpyrexia syndrome. Neurocrit Care 2009; 10: 136-40.

7) Kipps CM, Fung VS, Grattan-Smith P, de Moore GM, Morris JG: Movement disorder emergencies. Mov Disord 2005; 20: 322-34.

8) Nicholson D, Chiu W: Neuroleptic malignant syn- 
drome. Geriatrics 2004; 59: 36, 38-40.

9) American Psychiatric Association: Diagnostic and statistical manual of mental disorders: DSM-IV-TR. Washinton, American Psychiatric Association. 2000, pp 795-8.

10) Levenson JL: Neuroleptic malignant syndrome. Am J Psychiatry 1985; 142: 1137-45.

11) Pranzatelli MR: Oral pharmacotherapy for the movement disorders of cerebral palsy. J Child Neurol 1996; 11 Suppl 1: S13-22.

12) DeLong M, Wichmann T: Deep brain stimulation for movement and other neurologic disorders. Ann N Y Acad Sci 2012; 1265: 1-8.

13) Benabid AL, Benazzouz A, Hoffmann D, Limousin P, Krack P, Pollak P: Long-term electrical inhibition of deep brain targets in movement disorders. Mov Disord 1998; 13 Suppl 3: 119-25.

14) Vercueil L, Krack P, Pollak P: Results of deep brain stimulation for dystonia: a critical reappraisal. Mov Disord 2002; 17 Suppl 3: S89-93.

15) Counelis GJ, Simuni T, Forman MS, Jaggi JL, Tro- janowski JQ, Baltuch GH: Bilateral subthalamic nucleus deep brain stimulation for advanced PD: correlation of intraoperative MER and postoperative MRI with neuropathological findings. Mov Disord 2003; 18: 1062-5.

16) Urasaki E, Fukudome T, Hirose M, Nakane S, Matsuo H, Yamakawa Y: Neuroleptic malignant syndrome (parkinsonism-hyperpyrexia syndrome) after deep brain stimulation of the subthalamic nucleus. J Clin Neurosci 2013; 20: 740-1.

17) Perry D, Birthi P, Salles S, McDowell S: Neuroleptic malignant syndrome associated with the use of carbido$\mathrm{pa} /$ levodopa for dystonia in persons with cerebral palsy. PM R 2012; 4: 383-4.

18) Sakkas P, Davis JM, Janicak PG, Wang ZY: Drug treatment of the neuroleptic malignant syndrome. Psychopharmacol Bull 1991; 27: 381-4.

19) Tse L, Barr AM, Scarapicchia V, Vila-Rodriguez F: Neuroleptic malignant syndrome: a review from a clinically oriented perspective. Curr Neuropharmacol 2015; 13: 395-406. 